\title{
BIOMECHANICAL ANALYSES FOR THE EFFECTS OF ELBOW INITIAL FLEXION ANGLES ON UPPER EXTREMITY DURING A FALL
}

\author{
Pei-Hsi Chou', Shen-Kai Chen', You-Li ChOU ${ }^{2}$, Fong-ChIn Su ${ }^{2}$, \\ YUNG-CHIN SHI ${ }^{2}$, GWO-FENG HuANG ${ }^{2}$, TUN-CHIN Wu ${ }^{2}$ \\ ${ }^{1}$ Department of Orthopedic Surgery, Kaohsiung Medical University, \\ Kaohsiung, Taiwan \\ ${ }^{2}$ Institute of Biomedical Engineering, National Cheng-Kung University, \\ Tainan, Taiwan
}

\begin{abstract}
Elbow joint loading was evaluated during a forward fall at various elbow initial flexion angles, in order to determine which is the best elbow initial flexion angles to prevent the elbow injury during a fall. Subjects were asked to perform a forward fall and followed by a push-up motion in different elbow initial flexion angles: $0^{\circ}, 20^{\circ}, 40^{\circ}$ and unrestricted group. Fall on the outstretched hand is the leading cause of upper extremity injury. There are far more extension type of supra-condylar fracture of the elbow than flexion type. Flexion of the elbow may represent the effects of damper and spring. Using the motion analysis system, the kinematics and kinetics of the elbow joint were investigated under various elbow initial flexion angles. The loading biomechanics of the elbow joint differed with various elbow initial flexion angles. The ground reaction forces decrease with increase of elbow flexion upon impact. Different initial elbow flexion angles would affect the biomechanics of upper extremities during falls. Forward fall with elbow in extension is more dangerous. Knowledge of elbow kinematics and kinetics may be helpful in preventing injuries by reducing the ground reaction force with changes of the elbow initial flexion angles during a fall.
\end{abstract}

Biomed Eng Appl Basis Comm, 2002 (February); 14: 40-46.

Key words: Biomechanics, falls, elbow, shoulder, kinematics, kinetics, impact force

\section{INTRODUCTION}

Falls are common accidents in our daily life [1-4]. Accidental slips and falls in daily life, sports activity [5-8] or occupational environment [9] can result in trauma and functional disability of the upper extremity. Falls on the outstretched hand is the most common

Received: December 4, 2001; accepted: February 5, 2002 Correspondence: You-Li Chou, Professor

Institute of Biomedical Engineering, National Cheng Kung University, No.1 University Road, Tainan 701, Taiwan

E-mail: ylchou@mail.ncku.edu.tw cause of upper extremity injury, including approximately $90 \%$ of fractures at the distal radius, humeral neck, and supracondylar region the elbow $[10,11]$. In order to better prevent and manage these upper extremity injuries, there is a need to further understand the mechanisms of joint disorder in the elbow joint.

Approximately $75 \%$ of all fractures sustained by children occur in the upper extremities and frequently occur during a fall onto an outstretched hand. The majority of these injuries involve the wrist and forearm, but the elbow alone accounts for approximately 10 percent of all fractures in children [12]. Other clinical studies have also shown that falling on an outstretched hand plays an important role in the upper extremity 
trauma $[13,14]$. It is very important to understand the factors which influence fracture risk during a fall on the outstretched hand.

The study of Amis et al.[15] showed that the falling energy was mainly absorbed by muscles of upper extremities. The upper extremities played the roles of dampers. The two-mass-model of Chiu et al.[16] stated that the effective springs and effective dampers of wrist and shoulder can absorb the shock caused by impact. Nevertheless, they don't consider the flexion effect of elbow. Chou et al.[17] consider the effect further. Their result indicates that if the elbow flexes instead outstretches after impact would decrease the ground reaction forces. They suggest the elbow-flexed motion represents the effects of damper and spring. The three-mass-model of Chou then is more creative than Chiu's model.

The elbow-flexed motion during a fall is similar with the motion of pushup. The position of the hands during a pushup is important, however, the study of Lou et al.[18] shows that when the forearms internally rotate 45 degrees, the elbows may flex more easily, the study of Chou et al.[19] shows the same results.

Clinically, there are far more extension type of supra-condylar fractures of the elbow than flexion type. Furthermore, posterolateral instability of the elbow can be reproduced with forearm supination and mild elbow flexion. These evidences stress the importance of elbow flexion in the upper extremity trauma. Therefore, the purpose of this study is to investigate the effect of different initial elbow flexion angles on joint loading during a fall on the outstretched hand. To associate with prior studies in thinking, when forearm rotates internally, the elbow will then have an initial flexion angle. From this, we think whether different initial flexion angles of elbow at the moment of impact have various damping effects, and this is the motivation of the study. Purposes of the study are to investigate the effects of different initial flexion angle of elbow on ground reaction forces and impulses.

\section{METHODS}

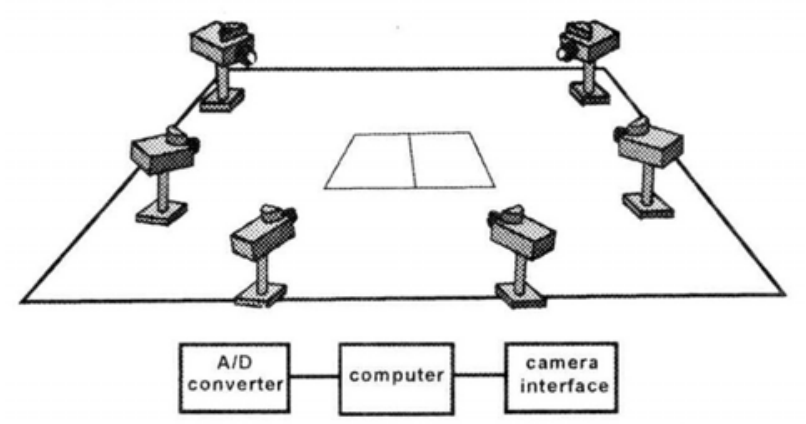

Fig. 1 The positions of 6 cameras and 2 force plates.

\subsection{Subjects and Experimental Protocol}

Twenty physically healthy male subjects volunteered for this investigation. They ranged from 23 to 26 years (mean 23.8, SD 2.0) of age, from 58 to $75 \mathrm{~kg}$ (mean 62.9, SD 6.1) in body weight, and from 168 to $176 \mathrm{~cm}$ (mean 171.7, SD 4.6) in body height. All subjects had no previous musculo-skeletal disorder of upper extremity, and all were right-hand dominant.

The ExpertVison motion system (Motion Analysis Corp., Santa Rosa, CA, USA) with six $120 \mathrm{~Hz}$ cameras and two $1000 \mathrm{~Hz}$ Kistler force-plates (Type 9281B, Kistler Instrument Corp., Winterhur, Switzerland) was used to measure relative joint positions and ground reaction forces (Fig.1).

A set of eleven reflective markers was placed on selected anatomic landmarks on the subject. The selected anatomic landmarks were intended to simulate the rigid body assumption for trunk (cervical vertebra 7 , thoracic vertebra 4 and acromion), upper arm (acromion process, medial and lateral epicondyles of the elbow), forearm (medial and lateral epicondyles of the elbow, ulnar styloid processes), and hand (radial and ulnar styloid processes, third metacarpal bone) (Fig. 2). In addition, a triangular frame with three-markers was

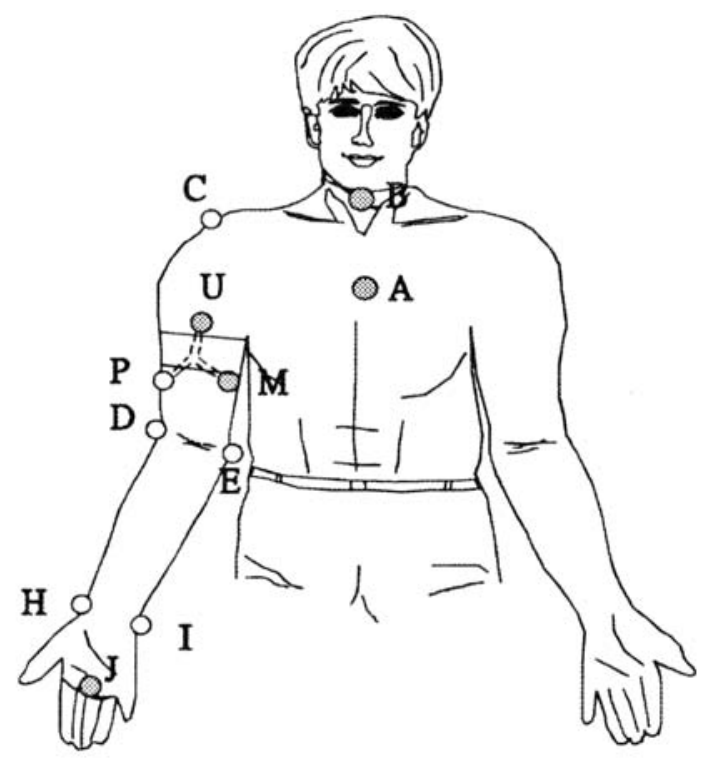

Fig. 2 Reflective markers:selected anatomic landmarks assume a rigid body for trunk.

$A$ : posterior surface of the chest $(\mathrm{T4})$

$B$ : spinous process of cervical vertebra (C7)

$C$ : acromion (postero-lateral border)

D : lateral epicondyle

E : medial epicondyle

$H$ : radial styloid process

I : ulnar styloid process

$\mathrm{J}:$ third metacarpal bone

$\mathbf{U} \cdot \mathbf{P} \cdot \mathbf{M}:$ Marker triad 
placed on the upper arm in order to minimize the potential errors due to skin movement of epicondyles during fall experiment. The shoulder joint center was defined by position starting from the elbow joint center, calculated by medial and lateral markers, to nearly $90 \%$ of the length from the elbow joint center to the marker at acromion.

In order to find out the relationship between initial elbow flexion and joint loading during a fall, four different elbow flexion angles $\left(0^{\circ}, 20^{\circ}, 40^{\circ}\right.$, unlimited) were tested in this study. Functional brace of the elbow was used to preset the different elbow flexion angles. During the testing, all subjects were asked to perform a forward fall, involving the following characteristics. First, the fall was directed forward, from a height of 5 $\mathrm{cm}$, and resulted in ground contact to the knees and a single outstretched hand. At the moment of impact the forearm is oriented at $15^{\circ}$ from the vertical. Second, ground contact occurred first to the knees and then the hand. After the impact, the body decelerated with a push-up motion. Furthermore, a questionnaire was also designed to record each subject's sensation of pain or discomfort during the impact. A score of 10 was the most comfortable, and a score of 0 was the most painful.

Three assumptions were made in this experiment: (1) each upper extremity segment was assumed a rigid body with constant density; (2) each joint was assumed to have three degrees of rotational freedom; (3) air friction and rope/pulley friction (of the safety restraint system) were neglected. The testing was approved by Orthopeadic and Rehabilitation Research Center, National Cheng-Kung University and by the National Science Council, Taiwan, for human subject testing. The consent form for this experiment was signed before performing the testing. At least the author or one of the coauthors with special sport medicine background stood by for the potential risk in the experiment.

\subsection{Theorem and mathematical equation}

Theorem of this study is based on Newton's second law of motion. Figure-3 shows the free body diagram of each segment. Base on Figure-3, substitute the distal force and distal moment of segment have known into the functions of Newton's second law of motion, we can get the proximal force and proximal moment of segments.

$\vec{F}_{h d}$ and $\vec{M}_{h d}$ are the distal force and distal moment of the hand, which can be measured by force plate. The proximal force and proximal moment of hand can then be calculated.

$\vec{F}_{h p}=m_{h} \vec{a}_{h}-\vec{F}_{h l}-m_{h} \vec{g}$

$\vec{M}_{h p}=I_{h} \vec{\alpha}_{h}+\vec{\omega}_{h} \times\left(I_{h} \vec{\omega}_{h}\right)-\vec{r}_{h p} \times \vec{F}_{h p}-\vec{M}_{h d}-\vec{r}_{h d} \times \vec{F}_{h d}$

$\vec{F}_{f d}$ is the reaction force of $\vec{F}_{h p}$, and $\vec{M}_{f d}$ is the reaction moment of $\vec{M}_{h p}$.

Substitute $\vec{F}_{f d}$ and $\vec{M}_{f d}$ into Newton's equation, the following equations can be determind.

$\vec{F}_{f p}=m_{f} \vec{a}_{f}-\vec{F}_{f d}-m_{f} \vec{g}$

$\vec{M}_{f p}=I_{f} \vec{\alpha}_{f}+\vec{\omega}_{f} \times\left(I_{f} \vec{\omega}_{f}\right)-\vec{r}_{f p} \times \vec{F}_{f p}-\vec{M}_{f d}-\vec{r}_{f d} \times \vec{F}_{f d}$

Similarly, $\vec{F}_{u d}$ is the reaction force of $\vec{F}_{f p}$, and

$\vec{M}_{u d}$ is the reaction moment of $\vec{M}_{f p}$. The following

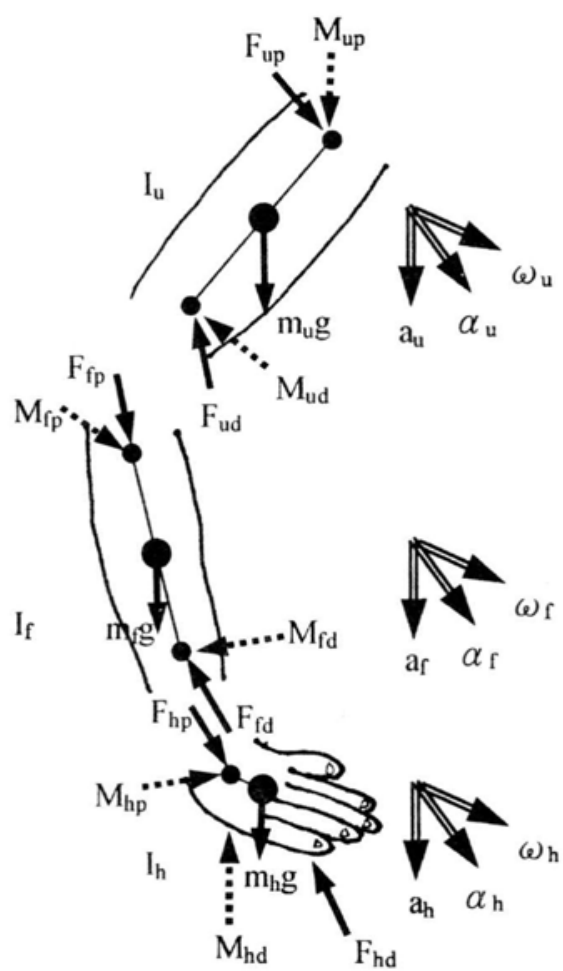

Fig.3 Free body diagram.
$F$ is force,
$M$ is moment,
$\mathrm{m}$ is mass,
I is moment inertia,
a is acceleration,
$\alpha$ is angular acceleration,
$\omega$ is angular velocity
$\mathrm{g}$ is gravity

in suffix
$\mathbf{h}$ is hand,
$\mathbf{f}$ is forearm
$\mathbf{u}$ is upperarm
$\mathbf{d}$ is distal
$\mathbf{p}$ is proximal




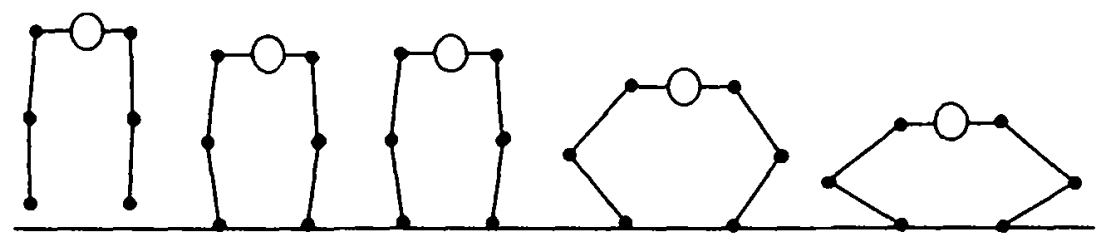

(1)

(2)

(3)

(4)

(5)

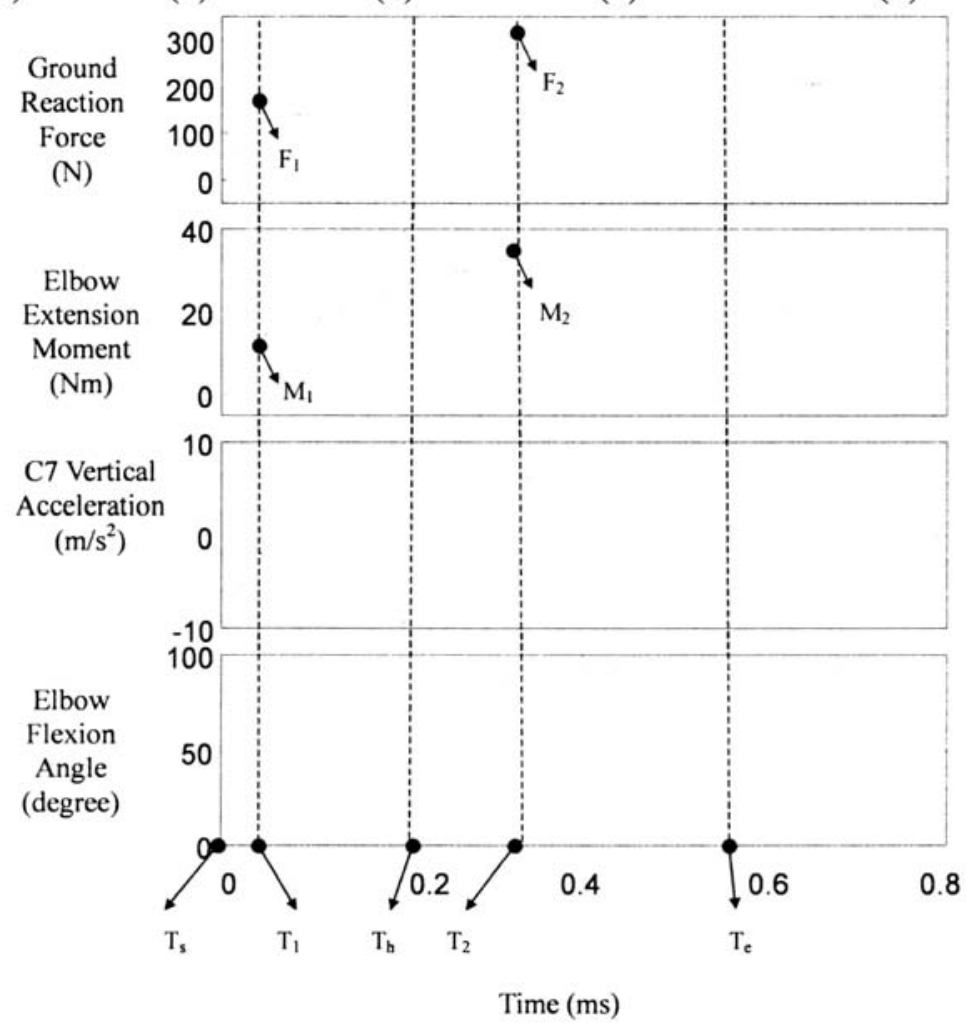

Fig. 4 Curves of biomechanical parameters

equations can be determind.

$$
\begin{aligned}
& \vec{F}_{u p}=m_{u} \vec{a}_{u}-\vec{F}_{u d}-m_{u} \vec{g} \\
& \vec{M}_{u p}=I_{u} \vec{\alpha}_{u}+\vec{\omega}_{u} \times\left(I_{u} \vec{\omega}_{u}\right)-\vec{r}_{u p} \times \vec{F}_{u p}-\vec{M}_{u d}-\vec{r}_{u d} \times \vec{F}_{u d}
\end{aligned}
$$

\subsection{Data Reduction}

Laboratory-developed kinematics and kinetics software were used to calculate the joint angles, resultant forces and moments of the elbow. A threesegment model, i.e. hand, forearm and upperarm, was employed in the analysis. Each segment was assumed to be a rigid body. Six CCD cameras were used to record the 3-D position of the markers. Three elbow joint angles, hinge angle, rotational angle and horizontal deviation were calculated using Euler's method with a $y-x-z$ rotational sequence based on the attached markers. A piezoelectric force plate was used to measure vertical and two shear forces as well as the location of the center of pressure of the palm on the plate and the moment about the axis normal to the force plate during the pushup. Simultaneous measurement of the upper-extremity kinematics was obtained by video recording of the markers. Segment mass and inertia data were estimated by anthropometry [20]. Angular velocity and acceleration were calculated with Euler's parametric method [21]. The force place loading equaled to the hand loading, with a reversed vector. The wrist loading was then calculated, using an inverse dynamic procedure with the Newton-Euler equations [21-23]. The loading of the elbow joints was then determined.

A generalized cross-validation spline smoothing (GCVSPL) routine with a cutoff frequency of $6 \mathrm{~Hz}$ and $15 \mathrm{~Hz}$ was used for data smoothing [24]. Joint angles and joint forces of the elbow as functions of temporal percentile during the impact period were calculated and then used for analysis [24]. 


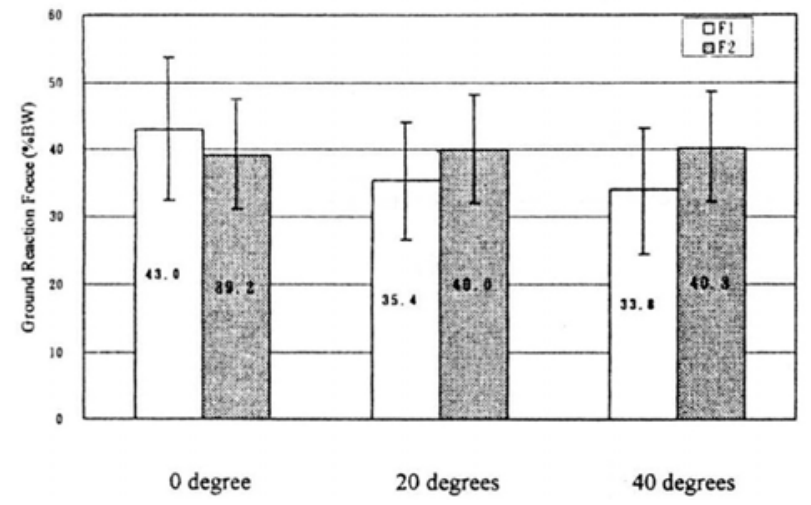

Fig. $5 \quad F_{1}$ and $F_{2}$ at various elbow initial flexion angles.

\subsection{Data Analysis}

Descriptive statistics were calculated for the ground reaction force, joints forces and joints moments. The ANOVA was used to test the hypotheses. Statistical analysis utilized the computer software SPSS7.0 for Windows with $\mathrm{P}<0.05$ as statistical significance.

\section{RESULT}

Curves in Fig. 4 are vertical ground reaction force (GRF), elbow extension moment, C7 vertical acceleration and elbow flexion angle. The GRF curve is twopeak type. After the impact, the first peak $F_{1}$ in the GRF curve is followed by the second peak $F_{2}$, and then the curve tends to steady. In the GRF curve. $T_{s}$ is defined as the point when GRF signal starts to occur; $T_{1}$ is defined as the point when the first peak $\left(F_{1}\right)$ occurs; $T_{2}$ is defined as the point when the second peak $\left(F_{2}\right)$ occurs; and $T_{e}$ is defined as the point when curve become to steady. Furthermore, the forces in this study are all normalized by body weight (\%BW).

1. Unrestricted group

The average initial flexion angle of this group was 31.0 (mean 31.0, SD 5.4) degrees, and the average pain score was 7.0.

2. Restricted groups

(1)Pain score

Mean score of the 0 degree group, 20 degrees group, and 40 degrees group were 6.2, 6.6, and 6.1 respectively.

(2)Ground reaction force

At the moment of impact, the $F_{1}$ was significantly different among various initial elbow flexion angles $(p<0.001)$ (Fig.5). The impact force $F_{1}$ decreased significantly with increased elbow flexion. Furthermore, $\mathrm{T}_{2}$ was significantly different among the various initial elbow flexion angle $(\mathrm{p}=0.001)$ (Fig.6). As a result, the time interval between $T_{1}$ and $T_{2}$ decreased with increased elbow flexion.

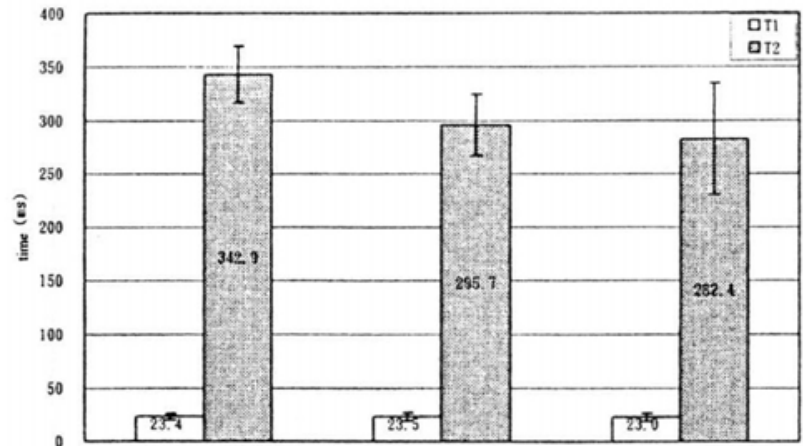

Fig. 6
$T_{1}$ and $T_{2}$ at various elbow initial flexion
angles.

(3)Elbow extension moment

$M_{1}$ and $M_{2}$ were defined as the moment of impact at $T_{1}$ and $T_{2}$ respectively (Fi.4). The $M_{1}$ were significant different among the three different elbow initial flexion angles $(p=0.002)$. But the $\mathrm{M}_{2}$ were not significant different among the three different elbow initial flexion angles ( $p=0.575)$ (Fig.7). The $M_{1}$ of the 0 degree group averaged (mean 10.4, SD 4.4) Nm. The $\mathrm{M}_{1}$ of the 20-degree group averaged (mean 12.7, SD 3.8) $\mathrm{Nm}$. The $\mathrm{M}_{1}$ of the 40-degree group averaged (mean 14.0, SD 4.7) $\mathrm{Nm}$.

The time of the second low frequency peak of $\mathrm{C} 7$ vertical acceleration, $F_{2}$ and $M_{2}$ in different elbow initial flexion angles groups did not differ significantly ( $p>0.177$ ). The elbow flexion angles at the time of $\mathrm{M}_{2}$ also did not show significant differences among the different initial elbow flexion $(p=0.706)$. The 0 degree group (mean 82.8, SD 9.8) degrees, 20 degrees group (mean 82.8, SD 6.3) degrees and 40 degrees group (mean 82.7, SD 10.6) degrees. The terminal elbow flexion angle at the time of $\mathrm{M}_{2}$ ranged form 70 to 100 degrees.

\section{DISCUSSION}

\subsection{Ground reaction force}

The curve of GRF of in this study is similar to that of Chiu [16]. The first high frequency peak occurred just after impact and followed by the second low frequency peak. However, they simulated their result as a two-mass-model, and did not consider the effect of elbow flexion. In their study, the subjects fell on the outstretched hand with elbow extended, and the dissipation energy produced by elbow moment was very small.

The results of Chou [19] showed that the first peak of GRF decreased with additional push-up motion after the impact. Their results also showed that the additional push-up motion could can dissipate much falling energy and decrease $F_{1}$ in effect. When elbow 


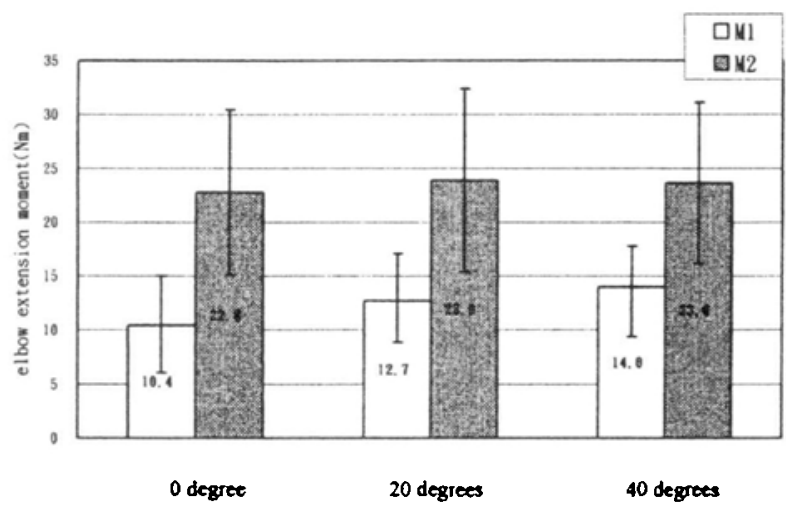

Fig. 7 Elbow extension moment at various initial flexion angles

flexes, the shoulder will be driven to extend, and this shoulder-extension also dissipates much falling energy. They suggest that the upper extremity plays the role of damper because of elbow flexion. In. In addition to the effective dampers and springs of wrist and shoulder, the elbow played a very important role of energy dissipation. Taking the effect of elbow motion into consideration, Chou's three-mass-model was more precise in simulating the motion of upper extremity during a fall.

In this study, the ground reaction force at impact $\left(F_{1}\right)$ decreased with increased elbow flexion upon impact. This elbow flexion effect could be used as a strategy in fall prevention technique, or designing an useful protective brace.

\subsection{Elbow extension moment}

The elbow extension moment $M_{1}$ was decreased when elbow initial flexion increased (Fig.7). If the elbow flexion angles at the time of $T_{1}$ were larger, the distance of force arm from the ground reaction force to elbow joint increased. Though the ground reaction force $F_{1}$ decreased with increasing elbow flexion, the effect of lever-arm is bigger than effect of $F_{1}$. Hence, $M_{1}$ increased with increased initial elbow flexion.

The ranges of flexion angles for $\mathrm{M}_{2}$ were from 70 to 100 degrees. In this ranges of flexion angles, the distance of force arm from ground reaction force to elbow joint was not different significantly. The time of the second low frequency peak of $F_{2}$ and $M_{2}$ in different elbow initial flexion angles group was not different significantly, and $\mathrm{F}_{2}$ was also not different significantly in different elbow initial flexion angles group. So, the effect of $M_{2}$ by different elbow flexion angles was small.

\subsection{Initial elbow flexion of 30 degrees}

According to the questionnaire, the pain score of the unrestricted group was the highest among the four groups; indicating that subjects preferred this initial flexion angle. The mean initial flexion angle of the unrestricted group was 30 degrees.

From the results of the three restricted groups, $\mathrm{F}_{1}$ and ground reaction impulse were significantly higher when the elbow was more extended. Although increased initial elbow flexion could reduce GRF $F_{1}$ and impulse, the subjects also feared of head injury from too much elbow flexion. Therefore, the subjects felt most comfortable when flexing their elbow to 30 degrees at the moment of impact.

\section{CONCLUSION}

The overall objectives of this study are to develop a biomechanical testing model for a simulated fall situation including various elbow initial flexion angles. The database established in this study will allow a better understanding of the mechanism in the upper extremity trauma. This testing model will be resulted in a strategy of preventing people from the injurie of the upper extremity during a fall.

In conclusion, the loading biomechanics of the elbow joint differs with various initial flexion angles. With the increasing elbow flexion at the moment of impact, the impact force (F1) decreases but the elbow extension moment (M1) increases. On the contrary, the impact force (F1) is at maximum when the elbow is fully extended upon impact. Therefore, a forward fall with mild elbow flexion maybe a better strategy to reduce impact force and to reduce injury.

\section{ACKNOWLEDGEMENTS}

The authors thank National Science Council on Taiwan for grant support (NSC 89-2314-B-037-102M08).

\section{REFERENCE}

1. Hoskin AF. Fatal falls: trends and characteristics. Statistical Bulletin MetropolitanInsurance Companies 1998; 79(2): 10-5.

2. Schneider EL, Guralnik JM. The aging of America. Impact on health care costs. Jama 1990; 263(17): 2335-40.

3. Melton LJI, Epidemiology of fractures, in Osteoporsis: Etiology, Diagnosis, and Management, B.L. Riggs, Melton, L.J. III, Editor. 1988, Raven Press: New York. p. 111-131.

4. Adesunkanmi AR, Oginni LM, Oyelami AO, Badru OS. Epidemiology of childhood injury. Journal of Trauma-Injury Infection \& Critical Care 1998; 44(3): 506-12.

5. Heller DR, Roultey V, Chamers S. Rollerblading injuries in young people. Journal of Paediatrics \& Child Health 1996; 32(1): 35-8.

6. Adesunkanmi AR, Oginni LM, Oyelami AO, 
Badru OS. Epidemiology of childhood injury. Journal of Trauma-Imjury Infection \& Critical Care 1998; 44(3): 506-12.

7. Manning DP, Ayers I, Jones C, Bruce M, Cohen K. The incidence of underfoot accidents during 1985 in a working population of 10,000 Merseyside people. Joural of Occupa-tional Accidents 1998; 10: 121-30.

8. Nevitt MC, Cummings SR. Type of fall and risk of hip and wrist fractures: the study of osteoporotic fracture. The Syudy of Osteoporotic Fractures Research Group [see comments]. Journal of the American Geriatrics Society 1993; 41(11): 1226-34.

9. Bentley TA, Haslam RA. Slip, trip and fall accidents occurring during the delivery of mail. Ergonomics 1998; $41(12)$ : 1859-72.

10. Melton LJ, Chao EYS, Lane J, Biomechanical aspects of fractures, in Osteoporosis:Etiology, Diagnosis, and Management. Raven Press, B.L. Riggs and L.J. Melton, Editors. 1998, Raven Press: New York. P. 111-31.

11. Fractures in Children. J.B. Lippencott, Philadephia, P.A, 1984.

12. Gill DR, Morrey BF. The Coonrad-Morrey total elbow arthroplasty in patients who have rheumatoid arthritis. A ten to fifteen-year follow-up study. Journal of Bone \& Joint Surgery - American Volume 1998; 80: 1327-135.

13. Leung KS, Kwan M, Wong J, Shen WY, Tsang A. Therapeutic functional bracing in upper limb fracture-dislocations. Journal of Orthopaedic Trauma 1988; 2: 308-13.

14. Kiwerski J. Peripheral nerve injuries associated with traumatic lesions of bones and joints of the upper extremity. Polski Przeglad Chirurgiczny 1972; 44: 1519-24.
15. Amis AA, Miller JH. The mechanisms of elbow fractures: an investigation using impact tests in vitro. Injury 1995; 26(3): 163-8.

16. Chiu J, Robinovitch SN. Prediction of upper extremity impact forces during falls on the outstretched hand. Journal of Biomechanics 1998; 31(12): 1169-76.

17. Chou YL, Chou PH, Lin CJ, Lin CF, Lou SZ. The Biomechanical Analysis of Impact Forces of Upper Extremity on a Fall. in Proceeding of Annual Symposium of Formosan Biomechanics Socity. 1999. Taipei, Taiwan.

18. Lou SZ, Lin CJ, Chou PH, Chou YL, Su FC. Elbow load during pushup at various forearm rotations. Clinical Biomechanics 2001; 16: 408-414.

19. Chou PH, Chou YL, Lin CJ, Chiu CF, Lou SZ, Su FC and Tan IC,"Effect of elbow flexion on Impact Forces of Upper Extremity during a Fall", Clini-cal Biomechanics. Vol.16/10, pp. 888-894, 2001.*SCI (Correspondence)

20. Dempster WT. Space requirements of the seated operator. OH: Wright-Patterson Air Force Base, Dayton, 1955: 55-159.

21. Haug EJ. Computer Aided Kinematics and Dynamics of Mechanical Systems Volume I: Basic Methods. Massachusetts: Allyn and Bacon, 1989:

22. Winter DA. Biomechanics and motor control of human movement. New York: John Wiley \& Sons, 1990: 75-102

23. OrthoTrak Full Body Gait Analysis-Reference Manual. California: Motion Analysis Corporation, 1993:

24. Woltring HJ. A FORTRAN package for generalized, cross-validatory spline smoothing and differentiation. Advances in Engineering Software 1986; 8: 104-13. 